\title{
Innovations and applications of the VERA quality control
}

\author{
D. Mayer, A. Steiner, and R. Steinacker \\ Department of Meteorology and Geophysics, University of Vienna, Vienna, Austria
}

Correspondence to: D. Mayer (dieter.mayer@univie.ac.at)

Received: 9 May 2012 - Published in Geosci. Instrum. Method. Data Syst. Discuss.: 24 May 2012

Revised: 27 August 2012 - Accepted: 4 September 2012 - Published: 8 October 2012

\begin{abstract}
Quality control (QC) is seen today as an important scientific field to increase the value of observational data. Whereas most QC methods are linked to atmospheric modeling (being part of the data assimilation procedure), in this paper the focus is on the application of a model independent QC method based on data self consistency recently published: VERA-QC. A special challenge is the QC of data in complex terrain which requires special treatment in terms of data selection and data transformation. In this context, some special VERA-QC modules such as the consideration of significant elevation differences of adjacent stations or the consideration of transformed temperature values will be discussed. The system detects gross errors as well as biases and offers objective correction proposals (deviations) for each observation. The essential gross error detection is not only based on the statistical behavior of station specific deviations, but also on the rate of cost function reduction. Beside a two dimensional application, higher dimensionalities may also be chosen, for instance including the time coordinate. Applications and results are discussed for pressure, temperature as well as for precipitation data which needs, however, a very dense observation network. Real time application of VERA-QC allows the production of high quality fields of meteorological parameters, which can be used, e.g. for nowcasting as well as for model unbiased validation of prognostic models.
\end{abstract}

\section{Introduction}

Nowadays, it is well known that the output of a numerical weather prediction (NWP) is, to a great extent, dependent on the quality of the input data. This awareness of the need for a high data quality evolved from the very beginning of the NWP era when the quality control (QC) of observations was still considered a purely technical task (Gandin, 1988). Within the last decades, many QC methods have been developed and it can be stated that a new research area was born. Taking that into account and considering the progressive international exchange of meteorological observations, the WMO started to set up a global standard regarding the quality of measurements in 1980. Different guidelines and manuals have been published and are updated on a regular basis (WMO, 1993, 2003, 2007, 2008).

High data quality is also essential when analyzing the actual state of the atmosphere, which constitutes the basis for a reliable nowcasting (Häggmark et al., 2000) or for model validation. Furthermore, the evaluation of long-term climatological data with respect to climate change, or the evaluation of climate models is utmost dependent on high quality data. If measurements are biased or error affected, they may be interpreted wrongly as a climate trend or may conceal existing climate signals (Haimberger, 2007).

The many different QC methods can be distinguished according to their usage of prior knowledge (such as error statistics) or model information (such as first guess fields). Some QC procedures are directly embedded in the data assimilation process of NWPs, while others are designed to be stand-alone preprocessing tools. Depending on the availability of single point observations or spatially and temporally distributed measurements, the applicable QC methods range from simple limit and plausibility checks to more sophisticated tests for spatiotemporal consistency. Steinacker et al. (2011) gives a detailed overview of the nowadays common QC methods and summarizes the different error types.

Leading European operational numerical weather prediction centers put a lot of effort in their data assimilation systems that provide the initial conditions for the analyses and forecasts of atmospheric compounds (e.g. European 
Centre for Medium-Range Weather Forecasts ECMWF, Rabier et al., 2000; UK's national weather service Met Office, Rawlins et al., 2007; French national meteorological service Météo-France, Fischer et al., 2005 or German meteorological service DWD, Wergen and Buchhold, 2002). The quality control of the used observations is part of the assimilation process (Andersson and Järvinen, 1999), but in most cases these observations have already been checked by preceding QCs carried out at the national institutions (e.g. Spengler, 2002). Smaller institutes usually do not run global models and therefore other methods are used to check the quality of their measurements. Salvati and Brambilla (2008) summarize the many different quality control procedures in Alpine meteorological services. Most of them can not afford the manpower to develop their own sophisticated QCs. This market niche has already been noticed and standalone QC routines can also be purchased (e.g. QualiMET used by DWD, Spengler, 2002 or the national meteorological and geophysical service of Austria ZAMG, Adler, 2009). Meanwhile, even open source QC-software has been developed and is distributed through the internet (e.g. kvoss, developed by the Norwegian Meteorological Institute (met.no) and the Swedish Meteorological and Hydrological Institute (SMHI)).

At the Department of Meteorology and Geophysics at the University of Vienna, an operational mesoscale analysis of basic meteorological parameters called VERA is carried out on an hourly basis. VERA is the abbreviation for Vienna Enhanced Resolution Analysis and describes a thin-plate spline interpolation method of irregularly distributed observations to a regular grid. A special feature of VERA is the possibility to combine measured values with measurement-independent a priori information called Fingerprints (Steinacker et al., 2006).

VERA analyses are used for teaching purposes at the department, for case studies, at field campaigns, for model validation or even for nowcasting at the Austrian Aeronautical Meteorology Service (Austro Control). In all these different fields of applications, the quality of the analyzed data is essential and a sophisticated preprocessing QC is required. Such a QC method has to be optionally independent of any prior knowledge, which is not available at field campaigns, and independent of model data, which would bias a model validation. Therefore, many existing advanced $\mathrm{QC}$ procedures (Bayesian QC, QC using optimum interpolation, variational QC) are not qualified for our purposes, and the performance of simpler spatial consistency checks (QC using spatial regression or inverse distance interpolation) was not convincing. An earlier QC procedure for VERA (Steinacker et al., 2000) was already based on the promising concept of minimizing the curvature of the analysis field. Nevertheless, its application was limited (e.g. to two dimensions, no cluster treatment, to a high degree depending on the station distribution) and hence, a new QC method had to be developed, named VERA-QC. The underlying mathematical concepts of VERA-QC, the recognition of gross errors, and a special treatment of clustered stations have been outlined in a previous paper (Steinacker et al., 2011). The scope of this follow-up article is to discuss the applicability of VERA-QC, its operational applications and improvements, as well as to present some case studies.

In Sect. 2 we present the available data which VERA-QC is applied to operationally, as well as an associated station selection algorithm using the concept of the minimum topography. Section 3 is concerned with summarizing the basic principles and special features of VERA-QC. Additionally, we discuss the applicability of VERA-QC which depends on the parameter under consideration and scale of phenomenon, as well as on the density of the observation network. The section is concluded by the presentation of recent innovations. Different types of case studies are presented in Sect. 4 in order to demonstrate the efficiency of the different QC modules and the overall performance of VERA-QC itself. In Sect. 5 we give a summary of further fields of applications which made use of VERA-QC, and Sect. 6 contains the conclusions.

\section{Data}

Every hour, approximately 2000 meteorological surface observations all over Europe are operationally combined to an analysis by VERA. Prior to that, they are checked by the two dimensional VERA-QC. The available data are gathered from SYNOP (surface synoptic observations) and METAR (Aviation routine weather reports) bulletins, as well as from the dense automatic station network of the Austrian Meteorological Service, but originate also from the Regional Meteorological Service of Friuli Venezia Giulia, OSMER (Osservatorio Meteorologico Regionale). The used parameters are precipitation RR, the zonal and meridional wind components $u$ and $v$, scalar wind speed $|\mathbf{V}|$, potential and equivalent potential temperature $\Theta$ and $\Theta_{\mathrm{e}}$ and mean sea level (MSL) pressure $p_{\text {red }}$. Note that in a preprocessing step $\Theta, \Theta_{\mathrm{e}}$ and $p_{\text {red }}$ are derived from the observed values for temperature, dew point and surface pressure. This step is necessary because different weather services may use different reduction formulas computing these parameters.

Supposing a two dimensional analysis of the actual ground observations (e.g. temperature measurements) in complex terrain, the predominant visible information is the height dependence. However, most applications require representative information regarding valleys and lowlands, and are not interested in the actual values at the mountain tops. Consequently, a station selection algorithm has been developed to exclude stations that are not representative for these regions. Therefore, the concept of the minimum topography is used. 
a

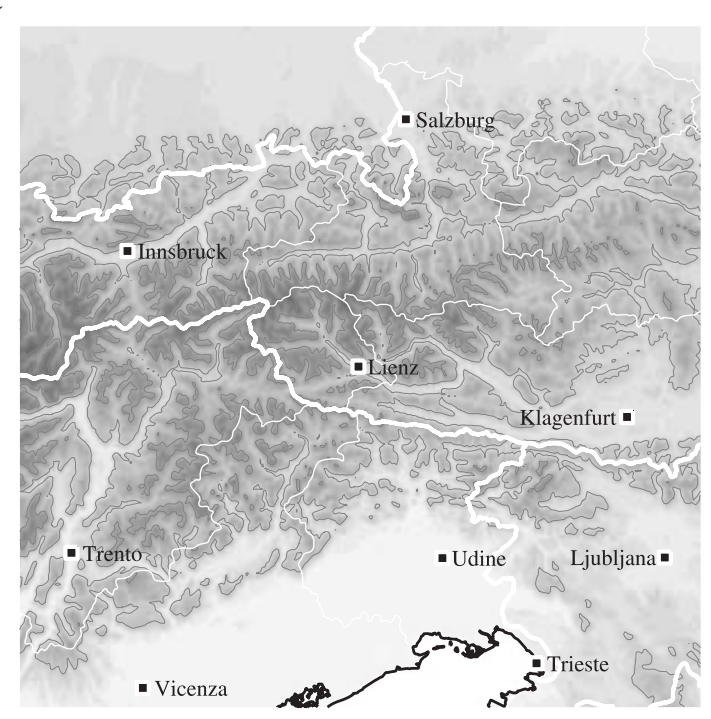

b

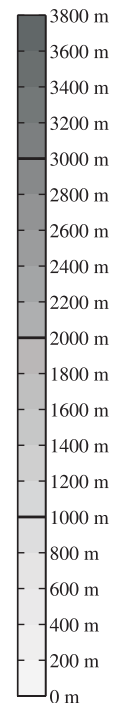

a

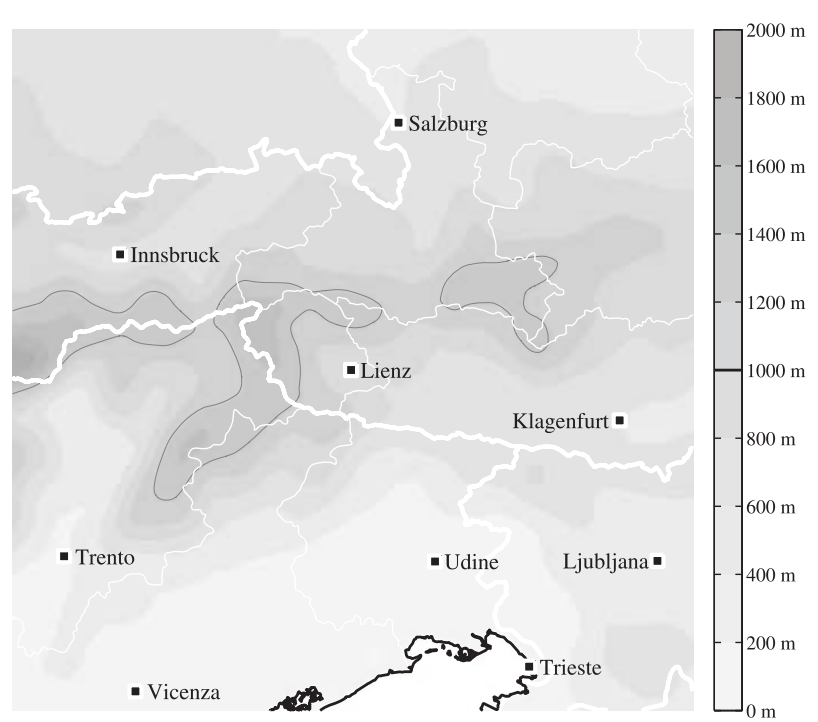

Fig. 1. Comparison of the real topography (a) and the idealized minimum topography (b) for the Central Alpine domain. The bold white lines illustrate national borders and thin white lines federal state borders. The black solid line in the south shows the coastline of the most northern part of the Mediterranean Sea. Major cities are marked by black squares with white frames. The shading refers to the absolute height above sea level.

\subsection{Minimum topography}

The minimum topography is derived from the real topography by smoothing summits and mountain ridges, but without lifting valleys, as conventional smoothing algorithms do. Plains are not affected by this modification, valleys and basins are widened, and the inclination of slopes is reduced, which may lead to the reduction of a mountain's elevation. Sufficiently narrow mountains between valleys disappear and, as a consequence, these valleys are connected to a broader artificial valley.

Mathematically, this procedure can be realized by replacing the real height at any point with the minimum height of all points within a certain (radial) distance from the considered point. This radius is typically set to a value of $10 \mathrm{~km}$ and the field obtained is smoothed in order to avoid rough structures.

In Fig. 1, the real topography (a) is compared to the idealized minimum topography (b) for a domain covering most of the Eastern Alps. Note that plains are not modified in (b) (e.g. the region between Vicenza and Udine), summits are smoothed (e.g. the main chain of the Alps), and only main valleys are preserved (e.g. Inn Valley with the city of Innsbruck, which is marked in the figure). For a more detailed example (Fig. 2), we consider a north-south cross section (held constant in east-west direction) through the mountains near Innsbruck. In addition to the height of the real topography (bold black solid line), the smoothed as well as the unsmoothed minimum topography is illustrated (bold gray solid line and thin gray solid line). The formerly mentioned properties of the minimum topography (smoothing summits,

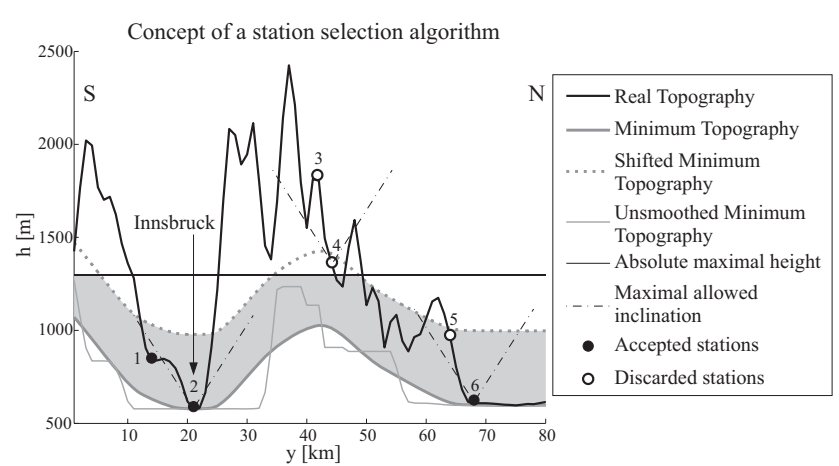

Fig. 2. Concept of the station selection algorithm on the basis of a north-south cross section through the mountains near Innsbruck (compare Fig. 1a). The reasons for accepting stations (compare stations 1,2 and 6) or for discarding stations (compare stations 3, 4 and 5) are explained in the text. Note that the absolute maximal height was set to $h=1300 \mathrm{~m}$ (instead of $h=1500 \mathrm{~m}$ ) and the ratio $r$ to $r=50 \mathrm{~m} \mathrm{~km}^{-1}$ (instead of $r=15 \mathrm{~m} \mathrm{~km}^{-1}$ ) in order to create an example containing all three criteria for excluding a station.

preserving main valleys, and leaving plains unmodified) are especially noticeable when comparing the real and the unsmoothed minimum topography.

This procedure is, for example, described in Bica et al. (2007). A similar approach is presented in Haiden (1998) where a comparable idealized topography is named valley floor surface. 


\subsection{Station selection algorithm}

There are three criteria for an observation to be excluded from an analysis:

- The absolute height $h$ of a station is too large. Computing reduced values (e.g. $p_{\text {red }}, \Theta, \Theta_{\mathrm{e}}$ ), a considerable reduction error can be expected for high stations. Therefore, the station selection algorithm of VERA-QC excludes all pressure observations above $h=1500 \mathrm{~m}$.

- The difference between the absolute height $h$ of a station and the height of the smoothed minimum topography $h_{\mathrm{MT}}$ exceeds $\Delta h=h-h_{\mathrm{MT}}=400 \mathrm{~m}$. Note that the vertical distance between valleys in the real topography and those mapped on the minimum topography can exceed a few $100 \mathrm{~m}$, depending on the inclination of the actual valley floor. This threshold is chosen in order to accept all stations along the valley floor, and, in contrast, to exclude stations that are located too high above the valley floor.

- If the ratio of the vertical and the horizontal distance $(r=\Delta z / \Delta x)$ between two stations is too large, the higher lying station is excluded. The user defined threshold for the maximal allowed ratio $r$ is defined as $r=15 \mathrm{~m} \mathrm{~km}^{-1}$. The meaning of this criterion becomes obvious when considering exposed wind measurements on hills or temperature measurements above an inversion. These observations would add a micrometeorological error to the analysis because they are not resolvable by the available observational network and therefore are excluded by this third regulation.

Note that, for a station to be excluded, only one criterion has to be fulfilled. Figure 2 illustrates an example station distribution and schematically outlines the different criteria of the station selection algorithm. Accepted stations are symbolized by black circles and discarded stations by white circles. Table 1 shows which regulations are, and which are not fulfilled by each single station.

Naturally, this station selection algorithm can be skipped or altered depending on individual requirements. Operationally, the VERA-QC (including this station selection algorithm) is optimized to deliver the most representative input data for VERA analyses.

\section{Methodology}

This section summarizes the basic concepts of VERA-QC, it shows which parameters the spatial consistency check can be applied to and it also presents innovations. The latter help to improve the QC of operationally collected data as well as of data collected in complex terrain.
Table 1. Decisions of the station selection algorithm for the six stations in Fig. 2. The columns labeled with Crit 1, Crit 2, and Crit 3 refer to the three criteria listed in Sect. 2.2 (absolute height $h$, difference $\Delta h$ between the absolute height $h$ and the height of the minimum topography $h_{\mathrm{MT}}$, and the ratio $r$ of the vertical and the horizontal distance between two stations). Accepted stations are marked by the $\sqrt{ }$ symbol and discarded stations by the $\times$ symbol.

\begin{tabular}{ccccc}
\hline Station & Crit 1 & Crit 2 & Crit 3 & Total \\
\hline 1 & $\sqrt{ }$ & $\sqrt{ }$ & $\sqrt{ }$ & $\sqrt{ }$ \\
2 & $\sqrt{ }$ & $\sqrt{ }$ & $\sqrt{ }$ & $\sqrt{ }$ \\
3 & $\times$ & $\times$ & $\times$ & $\times$ \\
4 & $\times$ & $\sqrt{ }$ & $\times$ & $\times$ \\
5 & $\sqrt{ }$ & $\sqrt{ }$ & $\times$ & $\times$ \\
6 & $\sqrt{ }$ & $\sqrt{ }$ & $\sqrt{ }$ & $\sqrt{ }$ \\
\hline
\end{tabular}

\subsection{Basic principles of VERA-QC}

Our previous article called Data Quality Control Based on Self-Consistency (Steinacker et al., 2011) was dedicated to explaining the mathematical background of VERA-QC in full detail. Subsequently, we give a short outline of these fundamentals.

After data passed simple limit checks, measurements of each parameter are examined for their spatial or, if requested, for their spatiotemporal self-consistency. For parameters $\Psi$ featuring a high redundancy with regard to the available observational network (e.g. $\Psi=\Theta$ or $p_{\text {red }}$ ), we postulate that the error-free analysis field $\Psi_{\mathrm{a}}$ should be smooth. Mathematically, this can be expressed as an optimization problem minimizing the cost function $J\left(\Psi_{\mathrm{a}}\right)$ which is defined as the sum of the squared curvatures $\mathcal{C}$ evaluated at the $n=1,2, \ldots, N(G)$ grid points:

$J\left(\Psi_{\mathrm{a}}\right)=\sum_{n}^{N(G)}\left(\mathcal{C}_{\Psi_{\mathrm{a}}}\right)_{n}^{2}=\sum_{n}^{N(G)} \sum_{d_{1}=1}^{D} \sum_{d_{2}=1}^{D}\left(\frac{\partial^{2} \Psi_{\mathrm{a}}}{\partial d_{1} \partial d_{2}}\right)_{n}^{2} \rightarrow \min$.

$d_{1}$ and $d_{2}$ denote the spatial or temporal coordinates of the considered $D$ dimensional domain, which are used for computing the curvature.

Due to the fact that neither the analysis field $\Psi_{\text {a }}$ nor its curvature are known, $\mathcal{C}\left(\Psi_{\mathrm{a}}\right)$ has to be approximated by a first order Taylor series around the curvature of the observed field $\mathcal{C}\left(\Psi_{\mathrm{o}}\right)$. Following this approach, we write the cost function $J$ as

$J=\sum_{m} \sum_{s} \sum_{d_{1}} \sum_{d_{2}}\left[\frac{\partial^{2} \Psi_{\mathrm{o}}}{\partial d_{1} \partial d_{2}}+\sum_{\mathrm{p}} \frac{\partial}{\partial \Psi_{\mathrm{p}}}\left(\frac{\partial^{2} \Psi_{\mathrm{o}}}{\partial d_{1} \partial d_{2}}\right) \Delta \Psi_{\mathrm{p}}\right]_{\mathrm{s}}^{2}$.

Before explaining Eq. (2) in more detail, we have to define the concept of local neighborhoods. In contrast to some common consistency checks, which work with a constant radius of influence, VERA-QC uses the concept of natural neighbors: with the help of the Delaunay triangulation, a primary 
neighborhood $(p(m)$, enclosing the first neighbors of the regarded central station $m$ and the central station itself) and a secondary neighborhood $(s(m)$, enclosing the neighbors of the primary stations, the primary stations as well as the regarded central station $m$ ) are defined for each station $m$. The advantage of this method is the possibility to adapt automatically to varying station densities within the whole considered domain.

These definitions in mind, Eq. (2) can be read as follows:

- The cost function $J$ consists of as many terms as stations exist in the considered domain (summation over $m)$.

- For each station, another summation over the corresponding secondary neighborhood (subscript $s$ ) is performed. The information for the Taylor series expansion related to the actually considered main station $m$ is gathered from this local secondary neighborhood.

- Note the subscript $p(p \in s)$, which denotes the primary neighborhood of the considered main station $m$. The values of these stations are allowed to vary; in other words, they are allowed to be erroneous.

- The differences $\left(\Psi_{\mathrm{a}}-\Psi_{\mathrm{o}}\right)=\Delta \Psi$ are the so-called deviations which present the only unknown variables in Eq. (2). By adding the deviations to the observation field, errors are corrected and the analysis field is obtained.

In order to compute these deviations, the cost function $J$ from Eq. (2) is differentiated with respect to all $\Psi_{m}$, which are the values of the analysis field at the positions of the $m=1, \ldots, N(\mathcal{M})$ stations in the considered domain. This leads to a system of linear equations that can be solved by matrix inversion.

\subsection{Special features of VERA-QC}

If the so calculated deviations are applied without further consideration, it could happen that an error propagates from one erroneous station to surrounding stations. The probability for such an error propagation is high in the case of gross errors, or if an erroneous station is located very close to others. In the following three subsections, we present a way to handle these problems.

\subsubsection{Reduction of the cost function}

Supposing an observation field with one central outlier, the VERA-QC would compute a high deviation for the error affected station, as well as smaller, but still significant deviations for the surrounding stations. In order to find out to what extent a deviation should be applied, its effect on the cost function $J$ is considered. A weighting factor $\mathcal{W}_{m}$ is obtained by applying only the deviation in question and observing the relative reduction of the analysis field's curvature. Weighting the deviations with $\mathcal{W}_{m}$ offers the advantage that deviations caused by the mentioned error propagation are reduced considerably.

\subsubsection{Recognition of gross errors}

These weights $\mathcal{W}_{m}$ are also used to recognize gross errors. If the cost function reduction exceeds a user defined threshold, and additionally, if the weighted deviation is large compared to all the other weighted deviations in the domain, the observation is assumed to present a gross error and therefore is discarded. After eliminating these gross errors, VERA-QC is repeated for the remaining stations.

Operationally, a cost function reduction of $80 \%$ is chosen to indicate a potential gross error. Additionally, the absolute value of the involved weighted deviation has to exceed the median of all absolute deviations within the whole domain by a certain factor (in the operational setting 30 times).

\subsubsection{Cluster treatment}

The concept of weighting with the cost function reduction usually works very well. Nevertheless, there are still some problems if a station, located very close to others, is erroneous. Therefore, close stations are combined to a fictive cluster station. At first, VERA-QC is applied to this modified station distribution. The computed weighted deviation for such a fictive cluster station is assigned and applied to the single cluster members. Then, VERA-QC is executed for the second time. Finally, the proposed corrections from both iterations are combined to a final deviation.

\subsection{Applicability of VERA-QC}

The spatial consistency check of VERA-QC is only applicable to measurements featuring a high degree of redundancy. This property is fulfilled if the scale of the considered phenomenon is large compared to the mean distance between the observation points. Looking at the available surface observation network and the investigated parameters (described in Sect. 2), the requirement of a high redundancy is not satisfied by the parameter precipitation and especially not by convective precipitation.

A method to quantify this criterion is to compare the mean station distance $\bar{d}$ to the parameter specific decorrelation distance $d_{0}$. For this purpose we consider hourly pressure and precipitation observations from June 2011. In order to compute the parameter specific decorrelation distances, all time series of observations were compared to each other and the corresponding correlation coefficients were calculated. In a further step they can be described by an analytic autocorrelation function $\rho(d)$. The decorrelation distance $d_{0}$ is defined as the distance where the autocorrelation function reaches the value $\rho\left(d_{0}\right)=e^{-1} \approx 0.37$. 

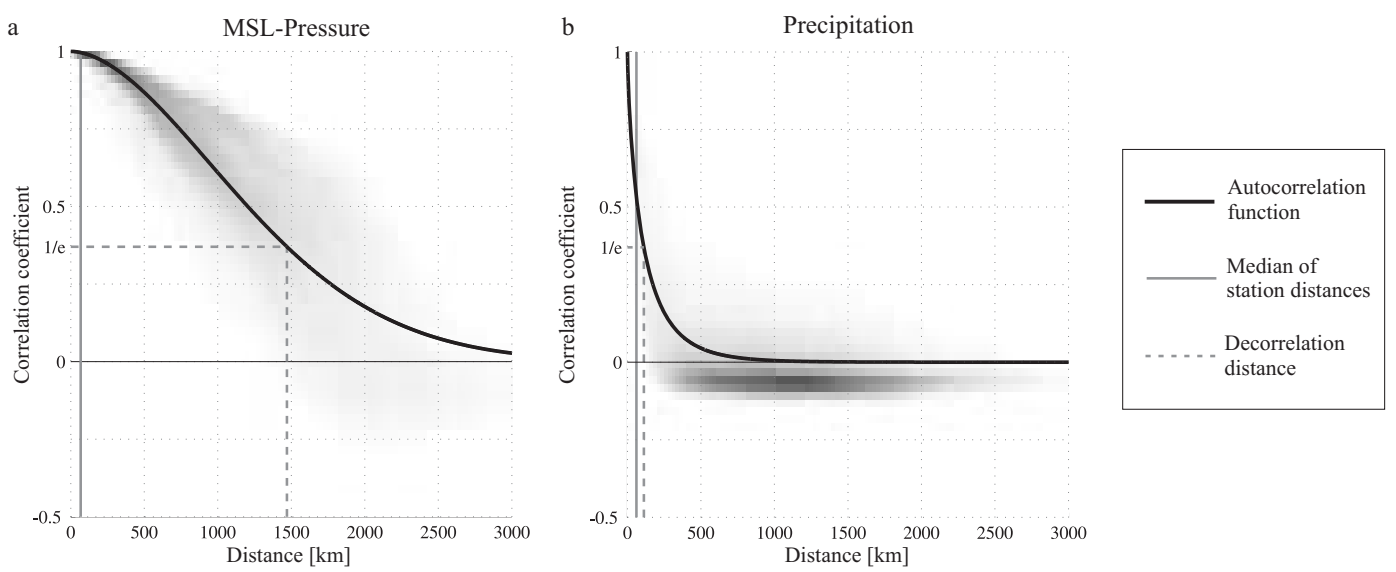

Fig. 3. Comparison of the autocorrelation for the parameters MSL-pressure (a) and precipitation (b) as a function of the station distances based on the hourly measurements of June 2011. The correlation is presented by the adjusted analytical autocorrelation function (black bold line). In addition to the decorrelation distance (gray dashed line), the median of all distances between neighboring stations is marked by a gray vertical line. Whereas in (a) the median of station distances is small compared to the decorrelation distance, in (b) they are of comparable magnitude.

Weber and Talkner (1993) give an overview of different autocorrelation functions of varying complexity. For our simple purpose, which is to compare the different spatial properties of precipitation and MSL-pressure, we chose the following exponential model as it is described, for example, in Gebremichael and Krajewski (2004):

$\rho(d)=\exp \left[-\left(\frac{d}{d_{0}}\right)^{s_{0}}\right]$.

The shape factor $s_{0}$ allows the autocorrelation function to differ from the common form of the exponential function. These two parameters $d_{0}$ and $s_{0}$ were adjusted with the help of a least square algorithm.

Figure 3 shows the autocorrelation as a function of the distance between all stations for the parameters MSLpressure (a) and precipitation (b). Instead of illustrating all the single scattered points $(d, \rho(d))$, the density of this point cloud is indicated by different shades of gray. In addition, the point cloud is described analytically by Eq. (3) and is symbolized by the black bold line. The dashed gray line indicates the decorrelation distance, whereas the vertical gray line illustrates the median of all distances between neighboring stations.

Note that, for adequately resolving a phenomenon, the mean distance between neighboring stations $\bar{d}$ has to be much smaller than the decorrelation distance $d_{0}$ of the considered parameter. As the two graphs in Fig. 3 show, the parameter MSL-pressure fairly fulfills this criterion $\left(\bar{d}=68 \mathrm{~km} \ll d_{0}=1475 \mathrm{~km}\right)$, precipitation, however, does $\operatorname{not}\left(\bar{d}=62 \mathrm{~km} \approx d_{0}=113 \mathrm{~km}\right)$.

It should be mentioned that the autocorrelation is not only a function of distance, but is also influenced by additional factors such as orography. Two (even more distant) stations at the same side of a main mountain ridge will most likely feature a higher autocorrelation than two stations separated by the mountain ridge (Lanzinger and Steinacker, 1990).

\subsection{Innovations of VERA-QC}

Since many observations in the operationally considered European domain are collected in complex terrain, special challenges regarding temperature measurements arise. Therefore, instead of considering potential temperature, the difference between $\Theta$ and the corresponding value of the standard atmosphere is controlled. Starting with the operational execution of VERA-QC, the deviations are stored continually and offer the possibility to compute a bias correction and are also used to improve the gross error recognition. In the following three subsections we will present these innovations.

\subsubsection{Consideration of relative values}

If the horizontal VERA-QC would be applied to temperature measurements, the temperatures of the few elevated stations would be increased significantly. In order to avoid this behavior, the potential temperature $\Theta$ is considered. Nevertheless, there still arises a problem. In general, the actual lapse rate is smaller than the dry-adiabatic lapse rate, but the computation of $\Theta$ bases on the assumption of a dry-adiabatic atmosphere. As a consequence, the 2-D VERA-QC systematically produces negative deviations for elevated stations as shown in Fig. 4. This behavior is more strongly pronounced in summer months due to the strong local heating in valleys (reduced air volume) and above elevated plains.

To overcome this problem, the relative value $\Theta_{\text {rel }}$, computed as the difference between $\Theta$ and the corresponding value of the standard atmosphere at station height $\Theta^{*}$, is calculated in a preprocessing step. This implicates the assumption that the actual lapse rate is approximated by the 


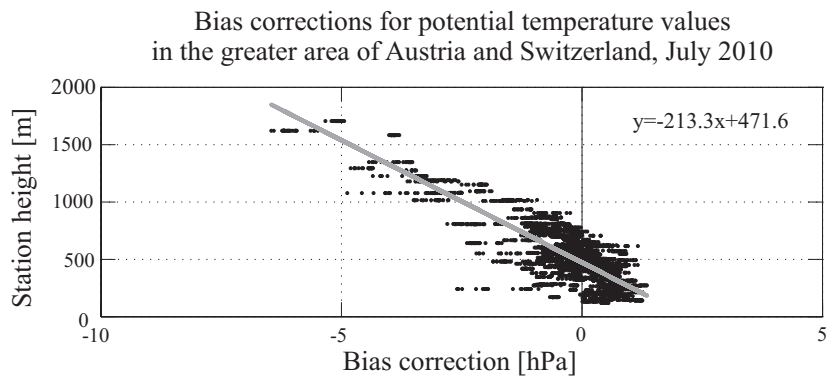

Fig. 4. Bias correction (black dots) for $\Theta$ as a function of the station height, evaluated for stations in the greater area of Austria and Switzerland in July 2010. The regression line (gray line) shows that the higher the stations, the more negative the bias corrections are. The absolute height limit of the station selection algorithm (operationally $1500 \mathrm{~m}$ ) was raised to $1800 \mathrm{~m}$ for this case study.

moist-adiabatic lapse rate $\left(\Gamma_{S}=0.0065 \mathrm{~K} \mathrm{~m}^{-1}\right)$, which is in accordance with the mean atmospheric conditions. The modified variable $\Theta_{\text {rel }}$ has the advantage to be distributed more smoothly even in complex terrain and a 2-D VERA-QC can be applied to $\Theta_{\text {rel }}$ without producing additional systematic errors.

Figure 5 illustrates the improvements that can be attributed to the consideration of the relative value $\Theta_{\text {rel }}$. The application of VERA-QC to $\Theta$ values yields the results presented in (a), whereas when applied to $\Theta_{\text {rel }}$ values (b), the systematic dependence on the height is reduced to a minimum.

For computing the equivalent potential temperature $\Theta_{\mathrm{e}}$, the lapse rate is already assumed to be the moist-adiabatic one. Thus, the described problem is not as pronounced for $\Theta_{\mathrm{e}}$ as it is for $\Theta$.

\subsubsection{Bias correction}

If a measurement of a single station is affected by a systematic error persisting in time (e.g. always yields in too high values), VERA-QC will compute deviations of the opposite sign (e.g. negative deviations) in order to correct the erroneous measurement. The operationally stored deviations for such a station are characterized by a median with absolute value significantly greater than zero. This shift in the deviations can be used to compute a bias correction.

The bias correction is defined as the median of the unweighted deviations $\Delta \Psi$ that have been collected within a parameter specific time interval. For the parameters $p_{\text {red, }}$, $u, v$, and $|\mathbf{V}|$, this time interval spans the last 30 days (720 hourly observations). The deviations for the parameters $\Theta$ and $\Theta_{e}$, as well as the parameters themselves, are subject to seasonal variations. Therefore, this time interval should span a whole year, but in order to save computational effort, the deviations of the last month and those of the month half a year ago are taken into account.

Figure 6 illustrates the chronological sequence of unweighted deviations for a station with bias affected pressure measurements. After 30 days, the first bias correction is computed and can be applied to the next day's measurements. Henceforward, at the end of each day, a new bias correction is computed. Note that the parameter specific time interval moves as well. As an example, the bias corrections for MSLpressure, valid for 31 July 2010, are shown in Fig. 7. As apparent in this figure, the bias corrections are spatially uncorrelated. Note the high magnitudes of the corrections, which are mainly due to reduction errors, caused by erroneous station heights.

Operationally, the bias correction is carried out at the beginning of VERA-QC. As a result, the roughness of the observation field is already reduced before minimizing the curvature of the analysis field. The deviations of the bias corrected measurements are rather distributed around zero and are mainly caused by random errors. If a systematic error featured a high magnitude, it often occurred that VERA-QC without bias correction identified the affected measurement to be a gross error. Thanks to the bias correction, observations of such stations are retained and increase the amount of utilizable data.

\subsubsection{Improvement of gross error detection}

The stored deviations can also be used for computing variable station specific thresholds to detect gross errors. These limits enhance the previously described gross error recognition, which is based on the impact a deviation has on the curvature of the analysis field (correcting a gross error leads to a significantly lower curvature, cf. Sect. 3.2.2).

Every month, new thresholds are computed based on the last month's weighted deviations. For the definition of a lower limit $\left(L_{1}\right)$ and an upper limit $\left(L_{2}\right)$, the first and the third quartile $\left(Q_{1}\right.$ and $\left.Q_{3}\right)$, as well as the interquartile range $\mathrm{IQR}=Q_{3}-Q_{1}$, are used:

$L_{1}=Q_{1}-3 \mathrm{IQR}, \quad L_{2}=Q_{3}+3 \mathrm{IQR}$.

An observation is identified as a gross error if its value does not fall within the interval $\left[L_{1}, L_{2}\right]$ and if, additionally, its absolute value exceeds a user defined constant limit. Note that, for the gross error recognition, only one criterion (the one described in this section or the one in Sect. 3.2.2) has to be fulfilled.

Figure 8 illustrates this concept for the equivalent potential temperature values of an exemplary station for November 2010 . The user defined constant limit is set to $\pm 4^{\circ} \mathrm{C}$ (thin solid black lines) for this parameter. Both thresholds (black dash dotted lines) are used in December 2010 for the gross error recognition.

If only the individual time series of measurements would be used for a station specific gross error detection based on statistical thresholds, extreme weather related observations are likely to be rejected by mistake. Using deviation time series instead offers additional spatial information to identify unusual atmospheric phenomena. 
a Median of unweighted deviations $\left[{ }^{\circ} \mathrm{C}\right]$ for $\Theta$, July 2010

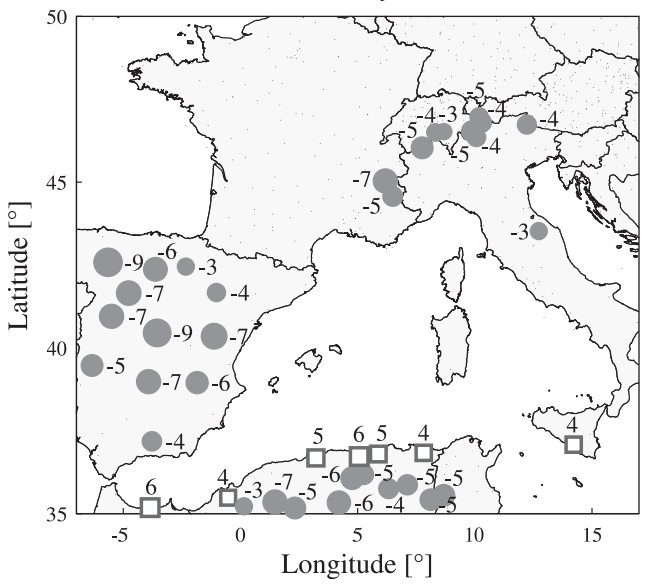

$\mathrm{b}$

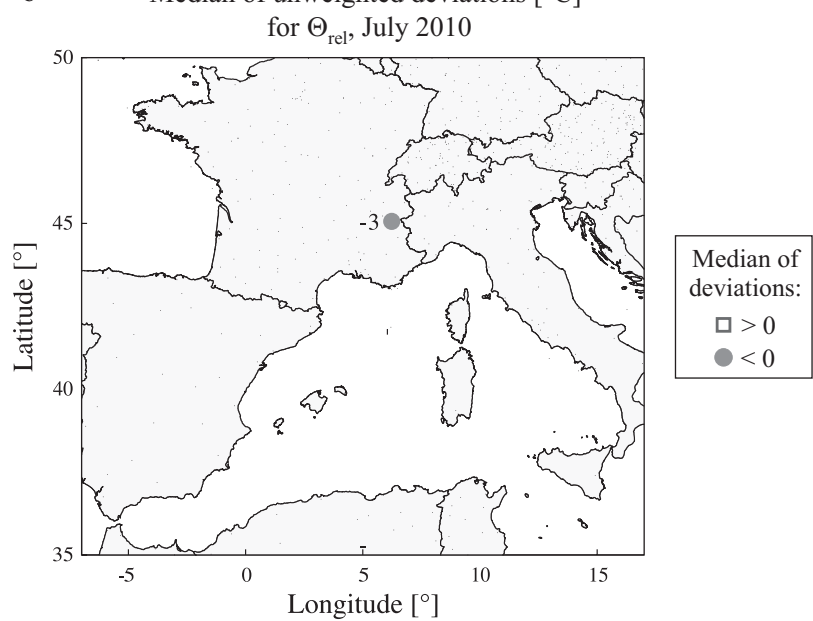

Fig. 5. Comparison of the median of unweighted deviations computed for $\Theta$ (a) and for the relative value $\Theta_{\text {rel }}=\Theta-\Theta^{*}$ (b) in July 2010 for a domain covering the Southwest Europe. Applying the VERA-QC to the potential temperature $\Theta$ (a), values of elevated stations are reduced systematically (circles), whereas values of neighboring coastal stations are increased (squares). If VERA-QC is applied to the difference between measurements and corresponding values of the standard atmosphere $\left(\Theta_{\text {rel }}\right)$, this height depending behavior is reduced significantly (b). For better visibility, only deviations of $|\Delta \Theta| \geq 3{ }^{\circ} \mathrm{C}$ are illustrated.

Computation of the bias correction

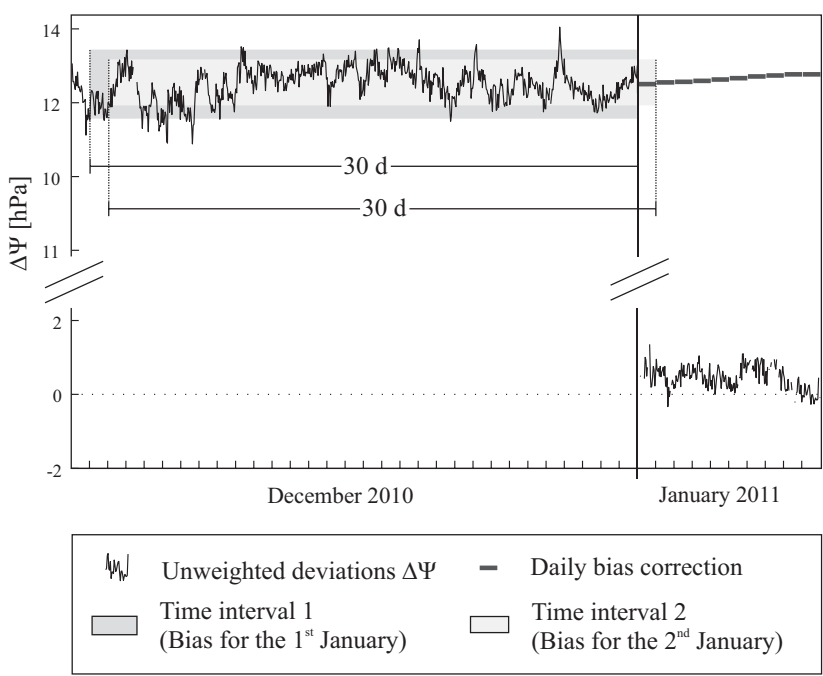

Fig. 6. Schematic illustration of computing a bias correction. The daily bias correction (dark solid horizontal bars starting at the beginning of January 2011) is defined as the median of the unweighted deviations (rough solid black line) within the user defined time interval (e.g. the last 30 days) and is valid for the next day. Note that the bias correction is allowed to vary and can automatically adapt to new situations (e.g. calibration of the instrument or changing station distributions).

Within the two preceding chapters, which describe the station selection algorithm and the methodology of VERA-QC, many different threshold values have been presented. The process of optimizing the chosen threshold values can be considered as an iterative process taking quite some time.
Bias correction for $\mathrm{p}_{\mathrm{red}}(>5[\mathrm{hPa}]), 31^{\text {st }}$ July 2010

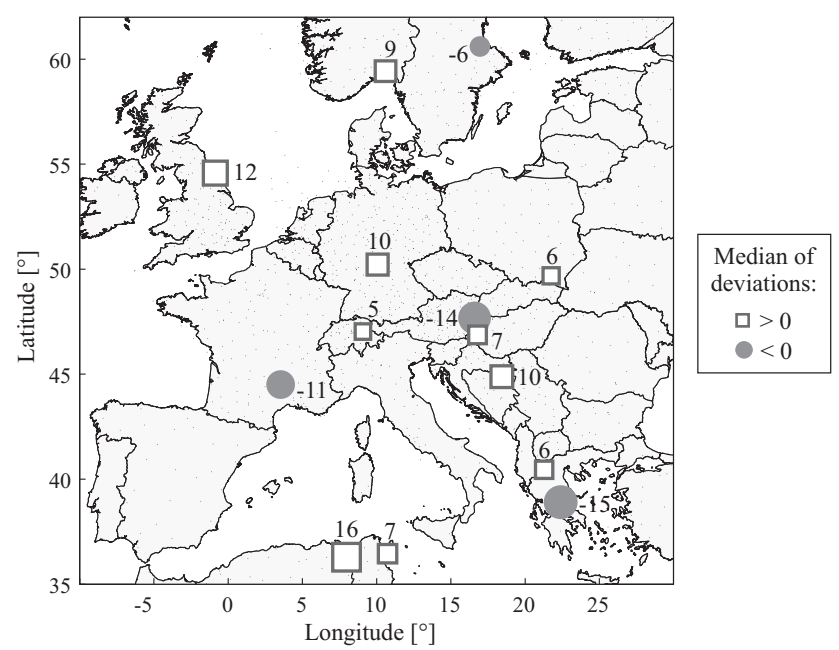

Fig. 7. Example spatial distribution of the bias corrections $>5 \mathrm{hPa}$ for MSL-pressure, valid for 31 July 2010 within the European domain. Circles symbolize negative bias corrections, and squares mark positive ones. In most cases, these can be explained by reduction errors (e.g. due to wrong station heights).

Experienced meteorologists supervise and evaluate the results achieved by a certain set of thresholds. According to their suggestions, the thresholds are altered. This procedure is being repeated constantly with the goal to optimize the proportion of rejected observations and that of accepted nonrepresentative observations for our available observational network (thus, for the available station network density). There are no objective configurations valid for an arbitrary 


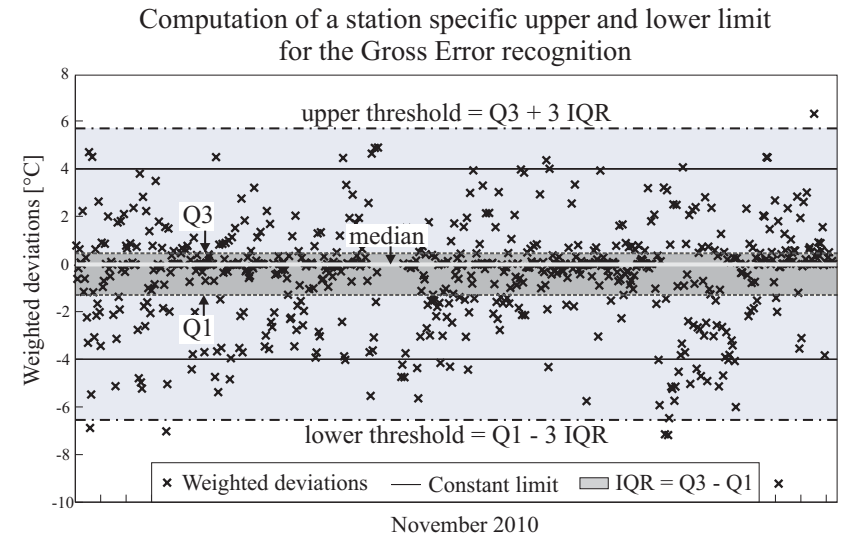

Fig. 8. Weighted deviations (black crosses) for $\Theta_{\mathrm{e}}$ of an exemplary station for November 2010. These constitute the basis for the computation of a variable station specific lower $\left(L_{1}\right)$ and an upper $\left(L_{2}\right)$ limit (black dash dotted lines), which support the gross error recognition. $Q_{1}$ and $Q_{3}$ denote the first and third quartiles (dark gray dashed lines) and IQR the interquartile range $\left(Q_{3}-Q_{1}\right.$, dark gray area). Additionally to the variable limits, constant values (thin solid black lines) of $\pm 4{ }^{\circ} \mathrm{C}$ have to be exceeded in order to recognize an observation as a gross error. In December 2010, all observations with weighted deviations outside the interval $\left[L_{1}, L_{2}\right]$ (light gray area) would be identified as gross errors.

observational network and the human component is essential at this point.

The setting of such thresholds is strongly constrained by the intended usage of observations and the relationship between hits, misses, false alarms and correct rejections has to be optimized individually. However, the intention of VERA$\mathrm{QC}$ is to provide representative measurements regarding the available observation network. This means, VERA-QC only accepts atmospheric phenomena of a scale that is large compared to the mean station distance.

Concluding this methodology chapter, it should be emphasized that the VERA-QC's strictness is depending on the local station density. In areas of a denser station network, VERA-QC can - with a higher reliability - control, confirm, or criticize measurements that were caused by phenomena of a wider range of meteorological scales. The probability of gross error detection for an error of the same magnitude decreases with increasing median station distance. This can be explained by the fact that an error of a certain magnitude leads in a dense observation network to a higher curvature of the observation field than it does in areas of lower station density. In order to quantify this relationship, we investigated the dependency of the absolute value of a deviation (identified as a gross error) on the median of station distances (considering only primary neighbors). Expressing this relationship in terms of a linear regression, we found a dependence of approximately $+3.5 \mathrm{~K} / 1000 \mathrm{~km}$ regarding potential temperature as an example (based on results from July 2011 to June 2012).

\section{Results and examples}

The following two sections present the changes and improvements visible in the VERA analysis if VERA-QC is used for preprocessing. First, monthly means of gridded hourly MSL-pressure values for July 2011 are considered. Second, the gradual improvements by applying different QC modules are investigated. In a third section, the 2-D and 3-D VERAQC is applied to a high-resolution precipitation data set. Real problems that arise when working with raw data collected at a field campaign are addressed.

\subsection{Effects of VERA-QC visible in mean values}

In order to investigate how the bias correction (Sect. 3.4.2) copes with systematic errors, monthly mean values of gridded hourly MSL-pressure observations are considered. This strategy was chosen because random errors are eliminated by averaging and thus do not distort the improvements caused by the bias correction.

After the hourly measurements of July 2011 have been analyzed with the help of the analysis tool VERA, these hourly gridded data were averaged. First, this procedure was applied to the pure, uncorrected observations (Fig. 9a) and secondly, to the quality controlled data (Fig. 9b). VERA-QC included all presented innovations, especially the bias correction.

In July 2011, the predominant pressure distribution was characterized by two lows (Baltic Sea and Aegean Sea) and a high pressure system (Bay of Biscay). Averaging the analyzed uncorrected observations, the resulting pressure patterns are mostly covered by systematic errors. In Fig. 9a, these errors are noticeable as locally persistent, mesoscale pressure perturbations. Some high-magnitude errors increase the curvature of the analysis field significantly.

When applying VERA-QC without bias correction to these observations, most of the systematic errors are recognized as gross errors and therefore are discarded. As a result, one would obtain an averaged field comparable to the one in Fig. 9b. But the advantage of the bias correction becomes obvious when investigating the amount of recognized gross errors. Whereas 9193 measurements $(0.99 \%)$ within July 2011 have been recognized as gross errors by VERA-QC without bias correction, this number has been reduced to 3522 $(0.38 \%)$ by applying the bias correction. Especially in areas with only few observations, no measurement should be discarded unnecessarily. Figure 9b (QC with bias correction) illustrates the predominant pressure patterns of July 2011 without troublesome noise.

\subsection{Step by step effects of VERA-QC}

The VERA-QC tool allows to activate or deactivate many different QC-modules as they were described in Sect. 3: the usage of the weighting factor, the recognition of gross errors, the cluster treatment, the consideration of relative values (for 
Monthly mean of the analyzed hourly pressure observations for July 2011

a

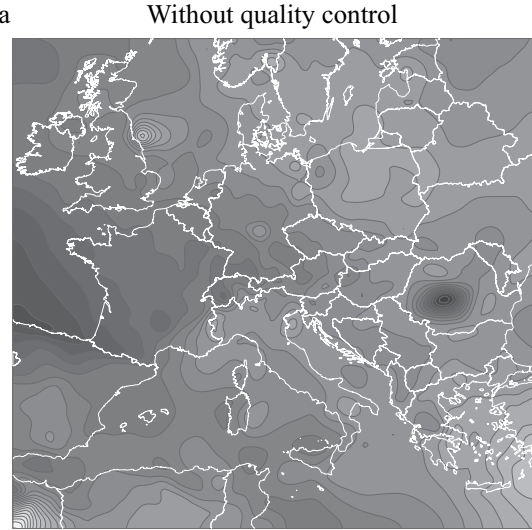

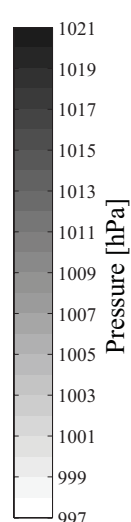

b With quality control including bias correction

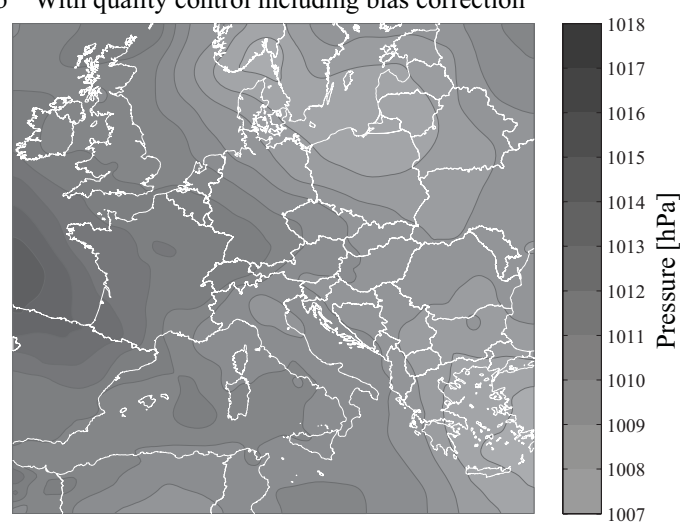

Fig. 9. Comparison of monthly mean values of the analyzed hourly MSL-pressure observations for July 2011 without QC (a) and with QC including bias correction (b). The hourly measurements have been analyzed with the help of the analysis tool VERA, the monthly mean values are based on the gridded data. Systematic errors, especially those with high magnitude (e.g. caused by reduction errors), have been corrected. As a result, the mean gridded values in (b) are distributed more smoothly.

potential temperature) and the bias correction. In Steinacker et al. (2011), we focused on presenting the effectiveness of the modules "usage of the weighting factors", "gross error recognition", and "cluster treatment". The "consideration of relative values" was the topic of Sect. 3.4.1 and in the following, the last module "bias correction" is investigated in more detail by means of a case study.

Conducting a field experiment or a case study, normally no prior knowledge to compute the bias correction is available and the corresponding bias correction module can not be executed. In order to present the quality achievable by VERA-QC with and without bias correction for one special case, Fig. 10 compares the original pressure observations of 5 July 2011, 06:00 UTC (a) to the quality controlled measurements after applying VERA-QC without (b) and with (c) activated bias correction. Note that both quality controlled fields (b) and (c) illustrate significant improvements compared to (a), and no essential difference between (b) and (c) is noticeable. Thus, the overall quality achievable for data collected at field campaigns or for case studies (in both cases no prior knowledge is available) is comparable to the quality achieved by a full QC run including bias correction. The advantage of a full VERA-QC, however, is that the amount of recognized gross errors and therefore rejected observations is reduced significantly (in the presented case: from $0.89 \%$ to $0.38 \%$ ). Depending on the individual requirements, it can be important to keep as many observations as possible. Climate studies, for instance, would intend to retain as many uninterrupted time series as possible and thus would profit from a bias correction.

\subsection{Usage of VERA-QC for field campaigns}

In order to present the versatility of VERA-QC and how it could be used at field campaigns or for case studies, we intend to focus on different possible applications of VERAQC to data, that were collected in 2007 at the field campaign COPS (Convective and Orographically-induced Precipitation Study, Wulfmeyer et al., 2011). We use measurements from the mesonet operated by the University of $\mathrm{Vi}$ enna, which was located in the Black Forest region in the South West of Germany. Ninety-six automatic weather stations (type HOBO) were arranged to resemble a regular grid with a mean distance between stations of approximately one kilometer. The high spatial and temporal resolution of observations $(\Delta x \approx \Delta y \approx 1 \mathrm{~km}, \Delta t=1 \mathrm{~min}$, but in the case of precipitation - due to the relatively low detection resolution $\Delta \mathrm{RR}=0.2 \mathrm{~mm}$ - accumulated to $\Delta t=10 \mathrm{~min}$ for postprocessing) allows to apply the 2-D or 3-D VERA-QC even to convective precipitation values as explained in Sect. 3.3. The considered time period ( $6 \mathrm{~h}$ from 18:00 to 24:00 UTC on 20 June 2007) was chosen because in this interval an intensive precipitation event occurred.

Depending on the particular requirements when using COPS data, an accurate QC has to fulfill certain specifications. One might be interested in keeping and therefore correcting as many observations as possible (minimal or no recognition of gross errors by the $\mathrm{QC}$ ) or one might allow the QC to eliminate suspicious measurements. Thus, the optimal settings for a $\mathrm{QC}$ depend on the types of errors the user wants to discard from the data set by all means (e.g. random-, gross- or systematic errors). In the following subsections, we will present three case studies outlining how the settings of VERA-QC can be adapted to different requirements. 


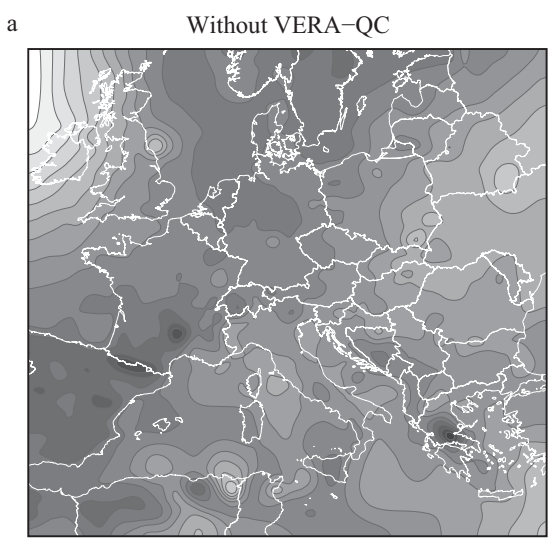

c VERA-QC (incl. GE recognition and bias correction)

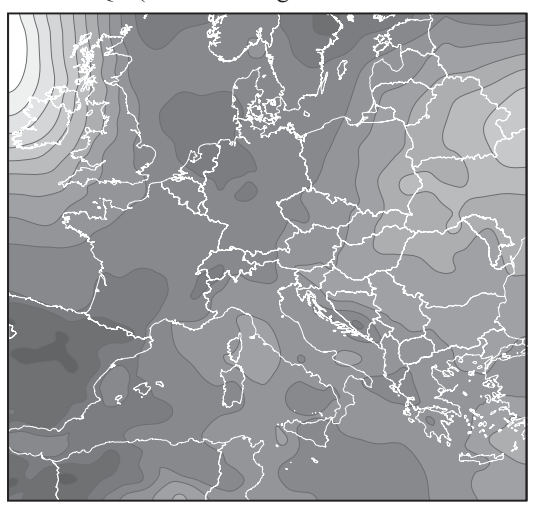

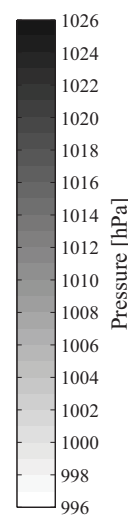

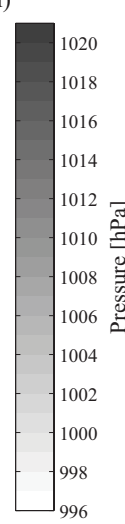

b VERA-QC (incl. GE recognition, no bias correction)

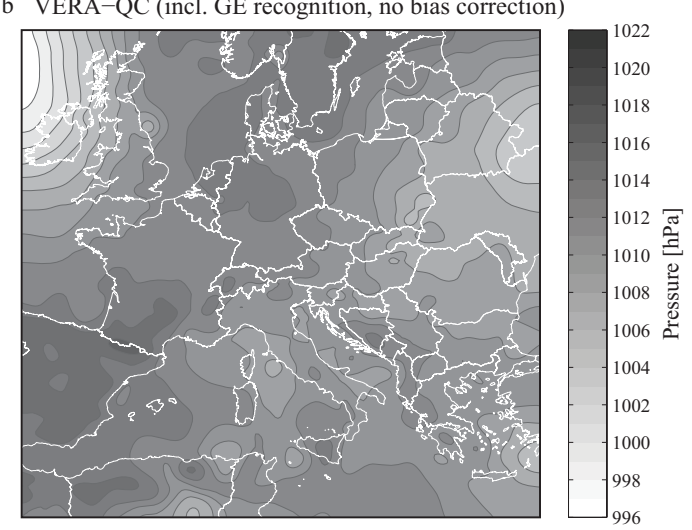

d Quality controlled minus original observations

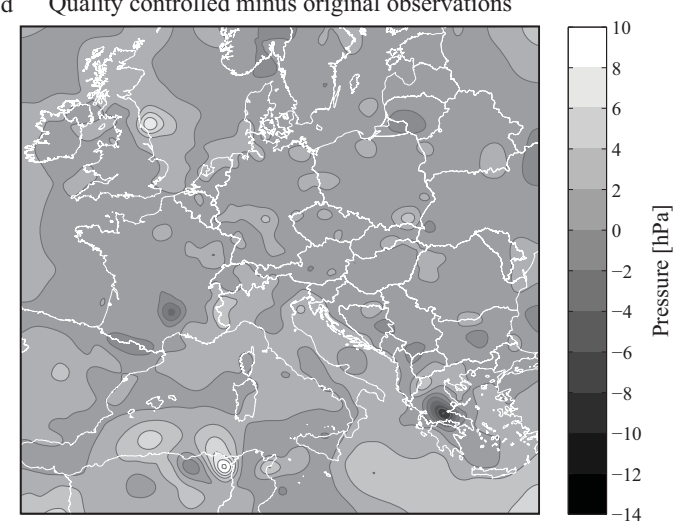

Fig. 10. Illustration of how observations are gradually improved by the VERA-QC. For all four figures, the values at the station positions in the European domain are interpolated to a regular grid by the analysis tool VERA. (a) Shows the original MSL-pressure values, computed from the 1567 observations on 5 July 2011, 06:00 UTC. (b) Presents the quality controlled observations, however no bias correction was applied. An amount of $0.89 \%$ of all observations are identified as gross errors and thus are excluded from the analysis. (c) Shows the results after applying the complete QC (with bias correction). The amount of gross errors is reduced to $0.38 \%$. Finally, in (d) the differences between the quality controlled and original observations are illustrated.

Note that, because of the regular station distribution, clustering was deactivated. Furthermore, the cost function reduction limit for detecting gross errors was set to $20 \%$ in all cases. Before controlling three dimensional $(x, y, t)$ data with VERA-QC, the spatial and temporal resolutions of observations get adjusted to be comparable (accounting for the typical speed of propagation of the considered phenomenon). This is done by multiplying the temporal coordinates by a so-called anisotropic factor, formally defined as a velocity. Additionally, this factor can also be used to weight the spatial or temporal coherence individually.

\subsubsection{QC of random tipping detected by rain gauges}

A well known problem when working with rain gauges is the random (temporally singular) erroneous registration of the minimal resolvable reading $(0.2 \mathrm{~mm})$ although no precipitation occurs. This random tipping may be caused by insects inside the gauge or by external forces shaking the instrument.

Figure 11 presents the observed 10 min precipitation values of 20 June 2007, 23:20 UTC. At this time two stations reported erroneous random tipping (light gray bars). Supposing the user does not allow to discard any observations, the gross error recognition module of VERA-QC was deactivated. Whereas the 2-D VERA-QC $(x, y)$ is able to correct the two erroneous observation values to an extent of $90 \%$, the 3 -D VERA-QC $(x, y, 18: 00 \leq t \leq 24: 00$ UTC) improves the percentage of correction to $98.5 \%$. The higher efficiency of a 3-D VERA-QC was already presented in Steinacker et al. (2011) by considering a comparable analytical example (see Fig. 9 of Steinacker et al., 2011). 


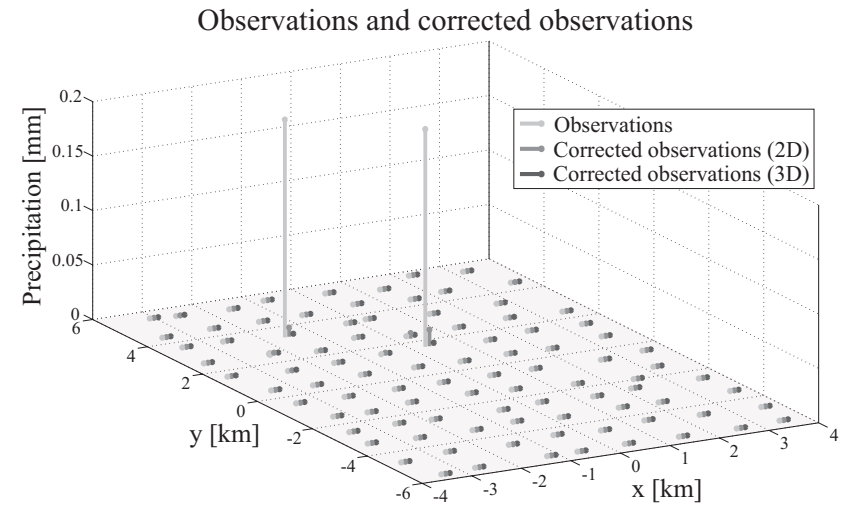

Fig. 11. Illustration of the station positions and observed 10 minutes precipitation values on 20 June 2007, 23:20 UTC, collected during the field campaign COPS (light gray bars). Note the two readings of $0.2 \mathrm{~mm}$ which are very likely due to erroneous random tipping or due to read out of memory, because no precipitation was observed at this time. The medium gray bars symbolize the observations corrected by a two dimensional $(x, y)$ VERA-QC without gross error recognition, and the dark gray bars stand for those corrected by a three dimensional $(x, y, t)$ VERA-QC without gross error recognition taking into account all measurements between 18:00 and 24:00 UTC.

\subsubsection{QC of a 2-D high resolution precipitation field}

When conducting measurements, it can always occur that one instrument fails. As in our case, we consider a rain gauge which registered constantly $0 \mathrm{~mm}$ independent of the occurrence of precipitation. Suppose doing a 2-D case study about a special precipitation event without having prior knowledge concerning previous or following measurements, one has to work, for example, with the information illustrated in Fig. 12 as light gray bars. At first sight, the observation marked by the black arrow seems to be erroneous. Applying the 2-D VERA-QC $(x, y)$ leads to the corrected observations, shown as dark gray bars in Fig. 12. The considered erroneous measurement was successfully adapted to its surrounding observations, which all registered a heavy precipitation event, even though the erroneous station is located at the edge of the observation field.

\subsubsection{QC of a systematic error without prior knowledge}

Although an erroneous instrument may in fact be able to register precipitation, it may occur that a temporally persistent constant value is added to the observations. Such a case is illustrated in Fig. 13. The measurements of the erroneous rain gauge are marked by black dots, the observations of 12 neighboring stations are symbolized by gray asterisks. Their comparison indicates a bias of $2 \mathrm{~mm}$ in $10 \mathrm{~min}$ for the former mentioned erroneous observation. If a case study demands to achieve a high accuracy, one has to accept that observations are flagged as gross errors and therefore are
Observations and corrected observations

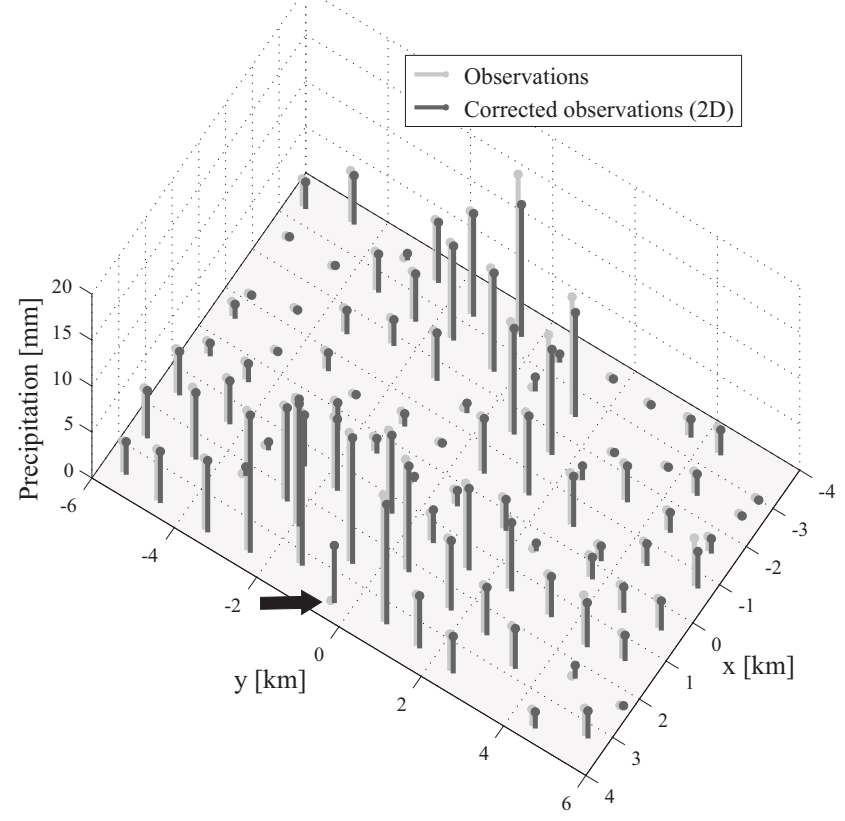

Fig. 12. Presentation of the observed $10 \mathrm{~min}$ precipitation values on 20 June 2007, 21:40 UTC as light gray bars. The dark gray bars mark the corrected observations after a two dimensional ( $x$, y) VERA-QC (without gross error recognition) was applied. The black arrow points at a station that was located within a field of heavy precipitation but nevertheless was stuck at a reading of zero millimeters. This malfunction could be observed for the entire period of 20 June 2007.

excluded. For the considered time interval (spanning 19:30 to 24:00 UTC) a 3-D VERA-QC $(x, y, t)$ with gross error recognition can be applied. In Fig. 13, the corrected observations are connected by the black and gray solid lines, respectively. Prior to and after the precipitation event centered at 22:00 UTC, the black solid line is interrupted, indicating that the biased observations were identified as gross errors and thus were excluded. Where no or only little precipitation was observed, the 3-D VERA-QC is able to correctly identify the biased measurement as a gross error. During the precipitation event, this erroneous observation is adjusted to the measurements in its surroundings. Although no bias correction was applied, the 3-D VERA-QC successfully handled this systematic error.

\section{Applications}

Quality controlled measurements constitute the basis for many different applications. Furthermore, also the computed deviations themselves offer valuable information. The following two sections overview different possibilities to make use of VERA-QC and its results. 


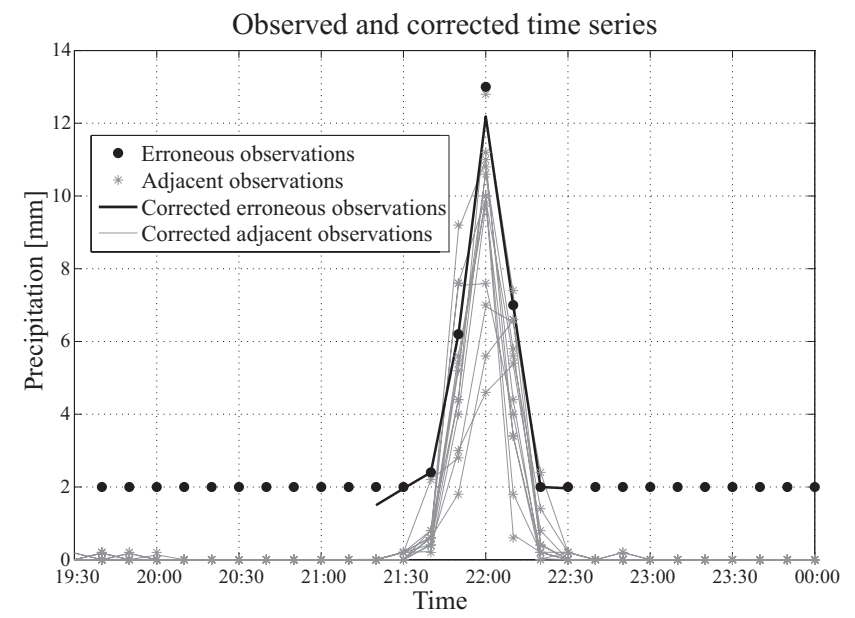

Fig. 13. Time series of observed (dots and asterisks) and corrected (solid lines) $10 \mathrm{~min}$ precipitation values on 20 June 2007. VERAQC was carried out including all three dimensions $(x, y, t)$. In this period, one station (symbolized by the black markers) constantly registered precipitation values of $2 \mathrm{~mm}$ even though no precipitation was observed in its surroundings (symbolized by the gray markers). Note that if the black line (presenting corrected values) is missing, the corresponding observation was identified to be a gross error and hence is excluded.

\subsection{Usage of quality controlled measurements}

At the department of Meteorology and Geophysics at the University of Vienna, several research projects have already been concerned with the application and improvement of VERA-QC. Many case studies have been conducted using different kinds of data sources, for example conventional GTS (Global Telecommunication Systems) data or even special observations taken at field experiments such as COPS. Another area of research within our department focuses on model verification. It is self-evident that high quality reference data are essential and VERA-QC was found to be able to provide these.

The most eminent application of VERA-QC is its usage as a preprocessing tool for the operational (hourly) analysis software VERA, which VERA-QC was named after. The so-called VERA-package (VERA-QC and VERA) delivers an exceptional performance and is therefore operationally used by the Austrian Aeronautical Meteorology Service (part of Austro Control) for nowcasting purposes. Currently, the VERA-package is also modified and prepared to be installed for operational use at the Swiss Federal Office of Meteorology and Climatology (MeteoSwiss).

\subsection{Usage of deviations computed by VERA-QC}

In Gorgas and Dorninger (2011), the idea of an analysis ensemble of deterministic, model-independent analyses is proposed. In the course of ensemble generation, the observations are manipulated using stochastic perturbations. One approach is to use the deviations computed by VERA-QC for determining the magnitude of these perturbations.

Investigating the temporal variations in the deviations helped Sperka and Steinacker (2011) to develop a method for creating a homogenized 3-hourly MSL-pressure analysis data set. After determining breaks in the deviations time series, the measurements within these breaks are corrected by the bias calculated from the deviations.

\section{Conclusions and final remarks}

As the mathematical concept of VERA-QC has already been described in Steinacker et al. (2011), this follow-up article is concerned with the operational application of the VERA-QC, innovations and the performance of the operational VERAQC demonstrated with the aid of case studies.

First of all, the applicability of VERA-QC to different meteorological parameters was discussed and we showed that, depending on the density of the observational network, even precipitation can be controlled by VERA-QC as demonstrated in the results section. Accounting for special difficulties in complex terrain, a station selection algorithm that excludes high-elevation stations that are not comparable to those in valleys and lowlands was introduced.

As a consequence of the operational application of VERA$\mathrm{QC}$, we had the possibility to develop new QC-modules. The collected deviations are used to continually compute a bias correction and variable station-specific limits, which support the gross error recognition. Additionally, the consideration of relative values helps to minimize the deviation's dependency on height, a problem that occurs especially in complex terrain.

In order to point out the positive influence of VERA-QC on a subsequent analysis, two case studies focus on the improvements achievable by using a bias correction as well as an enhanced gross error recognition. The possible application of VERA-QC to data collected at the field campaign COPS is discussed in more detail, including the use of a 3$\mathrm{D}(x, y, t)$ VERA-QC. In the concluding section, the many different fields of application of VERA-QC as well as of the computed deviations are summarized.

VERA-QC including the herein described innovations has been successfully carried out since January 2010 . The proposed corrections and hence the performance of VERA-QC are continually being inspected by experienced meteorologists. This human quality control of VERA-QC ensures an optimal performance and recognizes potential for improvements. One suggestion for the operational use is to weight the observations with a priori knowledge about the trustworthiness of a station's measurement, for example, primarily trust the observations taken by a well maintained station at an airport. Another idea is to compute the curvature and its derivations directly by fitting a second order polynomial surface through a station and its surroundings instead of computing the curvature by differentiating the interpolated field 
at local grid points. This would avoid using an inverse distance interpolation method for computing the values at the local grid points and, additionally, there would be no need to use finite differences for the numerical approximation when computing the curvature.

Concluding, we would like to advert to our web site http: //www.univie.ac.at/amk/veraflex/test/public/showing the latest VERA analyses for many different domains and parameters. Note that, by keeping the left mouse button pressed at a station symbol, not only the measured and corrected values used for the VERA analysis are displayed but also the weighted and unweighted deviations as well as the bias corrections. Furthermore, we point out that VERA-QC is made available for scientific use.

Acknowledgements. Thanks are due to the Austrian Science Fund (Fonds zur Förderung der wissenschaftlichen Forschung, FWF; P19658) and to the Austrian Research Funding Association (Die Österreichische Forschungsförderungsgesellschaft, FFG; project 818110) for partial financial support of this work.

Edited by: M. Genzer

\section{References}

Adler, S.: Datenkontrolle der ZAMG mit dem Prüfprogramm QUALIMET von der Firma Ernst Basler \& Partner, internal report, Vienna, Austria, 2009.

Andersson, E. and Järvinen, H.: Variational quality control. Q. J. Roy. Meteorol. Soc., 125, 697-722, 1999.

Bica, B., Steinacker, R., Lotteraner, C., and Suklitsch, M.: A new concept for high resolution temperature analysis over complex terrain, Theor. Appl. Climatol., 90, 173-183, 2007.

Fischer, C., Montmerle, T., Berre, L., Auger, L., and Stefanescu, S. E.: An overview of the variational assimilation in the ALADIN/France numerical weather-prediction system, Q. J. Roy. Meteorol. Soc., 131, 3477-3492, 2005.

Gandin, L. S.: Complex Quality Control of Meteorological Observations, Mon. Weather Rev., 116, 1137-1156, 1988.

Gebremichael, M. and Krajewski, W. F.: Assessment of the Statistical Characterization of Small-Scale Rainfall Variability from Radar: Analysis of TRMM Ground Validation Datasets, J. Appl. Meteorol., 43, 1180-1199, 2004.

Gorgas, T. and Dorninger, M.: Concepts for a pattern-oriented analysis ensemble based on observational uncertainties, Q. J. Roy. Meteorol. Soc., 138, 769-784, 2011.

Häggmark, L., Ivarsson, K., Gollvik, S., and Olofsson, P.: Mesan, an operational mesoscale analysis system, Tellus A, 52, 2-20, 2000.

Haiden, T.: Analytical aspects of mixed-layer growth in complex terrain. Preprints, Eighth Conference on Mountain Meteorology, Amer. Meteor. Soc., Flagstaff, Arizona, 368-372, 1998.

Haimberger, L.: Homogenization of Radiosonde Temperature Time Series Using Innovation Statistics, J. Climate, 20, 1377-1403, 2007.

Lanzinger, A. and Steinacker, R.: A Fine Mesh Analysis Scheme Designed for Mountainous Terrain, Meteorol. Atmos. Phys., 43, 213-219, 1990
Norwegian Meterological Institute: kvalobs open source software project (kvqc2d, version: 1.3.1), an Open Source Software for the Quality Control of Geophysical Observations, http://kvalobs. wiki.met.no (last access: 4 October 2012), 2011.

Rabier, F., Järvinen, H., Klinker, E., Mahfouf, J.-F., and Simmons, A.: The ECMWF operational implementation of fourdimensional variational assimilation, I: Experimental results with simplified physics, Q. J. Roy. Meteorol. Soc., 126, 1143-1170, 2000

Rawlins, F., Ballard, S. P., Bovis, K. J., Clayton, A. M., Li, D., Inverarity, G. W., Lorenc, A. C., and Payne, T. J.: The Met Office global four-dimensional variational data assimilation scheme, Q. J. Roy. Meteorol. Soc., 133, 347-362, 2007.

Salvati, M. and Brambilla, E.: Data quality control procedures in Alpine meteorological services, Tech. rep., Regional Agency for Environmental Protection of Lombardia, Università degli Studi di Trento Dipartimento di Ingegneria Civile e Ambientale, 2008.

Spengler, R.: The new Quality Control- and Monitoring System of the Deutscher Wetterdienst, WMO Techn. Conf. on Meteorol. and Environm. Instrum. and Methods of Observ., Bratislava, 2002.

Sperka, S. and Steinacker, R.: A Quality-Control and BiasCorrection Method Developed for Irregularly Spaced Time Series of Observational Pressure Data, J. Atmos. Ocean. Tech., 28, 1317-1323, 2011

Steinacker, R., Häberli, C., and Pöttschacher, W.: A Transparent Method for the Analysis and Quality Evaluation of Irregularly Distributed and Noisy Observational Data, Mon. Weather Rev., 128, 2303-2316, 2000.

Steinacker, R., Ratheiser, M., Bica, B., Chimani, B., Dorninger, M., Gepp, W., Lotteraner, C., Schneider, S., and Tschannett, S.: A Mesoscale Data Analysis and Downscaling Method over Complex Terrain, Mon. Weather Rev., 134, 2758-2771, 2006.

Steinacker, R., Mayer, D., and Steiner, A.: Data Quality Control Based on Self-Consistency, Mon. Weather Rev., 139, 3974-3991, 2011.

Weber, R. O. and Talkner, P.: Some Remarks on Spatial Correlation Function Models, Mon. Weather Rev., 121, 2611-2617, 1993.

Wergen, W. and Buchhold, M.: Datenassimilation für das Globalmodell GME, Promet, 27, 150-155, 2002.

WMO: Guide on the Global Data-processing System, World Meteorological Organization, WMO (Series), no. 305, 1993d Edn., http://www.wmo.int/e-catalog/detail_en.php?PUB_ID= 380\&SORT $=$ N\&q= (last access: 4 October 2012), 1993.

WMO: Manual on the Global Oberving System, World Meteorological Organization, WMO (Series), no. 544, 2003d Edn., www. wmo.int/pages/prog/www/OSY/Manual/WMO544.pdf (last access: 4 October 2012), 2003.

WMO: Guide to the Global Observing System, World Meteorological Organization, WMO (Series), no. 488, 2007th Edn., http://www.wmo.int/pages/prog/www/OSY/Manual/488 Guide_2007.pdf (last access: 4 October 2012), 2007.

WMO: Guide to Meteorological Instruments and Methods of Observation, World Meteorological Organization, WMO No. 8, 7th Edn., http://www.wmo.int/pages/prog/gcos/documents/ gruanmanuals/CIMO/CIMO_Guide-7th_Edition-2008.pdf (last access: 4 October 2012), 2008. 
Wulfmeyer, V., Behrendt, A., Kottmeier, C., Corsmeier, U., Barthlott, C., Craig, G., Hagen, M., Althausen, D., Aoshima, F., Arpagaus, M., Bauer, H., Bennett, L., Blyth, A., Brandau, C., Champollion, C., Crewell, S., Dick, G., Di Girolamo, P., Dorninger, M., Dufournet, Y., Eigenmann, R., Engelmann, R., Flamant, C., Foken, T., Gorgas, T., Grzeschik, M., Handwerker, J., Hauck, C., Höller, H., Junkermann, W., Kalthoff, N., Kiemle, C., Klink, S., König, M., Krauss, L., Long, C., Madonna, F.,
Mobbs, S., Neininger, B., Pal, S., Peters, G., Pigeon, G., Richard, E., Rotach, M., Russchenberg, H., Schwitalla, T., Smith, V., Steinacker, R., Trentmann, J., Turner, D., van Baelen, J., Vogt, S., Volkert, H., Weckwerth, T., Wernli, H., Wieser, A., and Wirth, M.: The Convective and Orographically-induced Precipitation Study (COPS): the scientific strategy, the field phase, and research highlights, Q. J. Roy. Meteorol. Soc., 137, 3-30, 2011. 